This is the final author version of the article:

White, W. and Hemmings, C. (2010) KULTUR: showcasing art through institutional repositories, Art Libraries Journal, 35 (3), 30-34

\title{
KULTUR: showcasing art through institutional repositories
}

\author{
Wendy White, Faculty Librarian and Institutional Repository \\ Manager, University of Southampton whw@soton.ac.uk \\ Clare Hemmings, Liaison Librarian, Winchester School of Art \\ Library, University of Southampton ch1@soton.ac.uk
}

\begin{abstract}
Showcasing work has always been at the heart of the arts community, whether it be through an exhibition, site-specific installation or performance. Representation of the original work has also been important and use of print-based options like exhibition catalogues is now complemented by use of websites and multi-media friendly services like Flickr and YouTube and Vimeo. These services also provide options for sharing born-digital material. For those working in higher education there is a need to profile both the personal and the institutional aspects of creative outputs. The KULTUR project created a model for arts-based institutional repositories and it is hoped that this approach will be useful for other arts institutions.
\end{abstract}

\section{The Project}

The first institutional repositories (IRs) tended to focus on the public dissemination of text-based work, including articles, reports, theses and conference material. Before the start of the Kultur project none of the UK's specialist higher education arts institutions had an institutional repository and other broad-based institutions had limited content from their Schools of Art. The aim of the JISC funded KULTUR project was to use the EPrints software to develop a repository that was especially suited to the needs of the arts community. The repository would be designed specifically to showcase visual and timebased work and complex digital objects. The project would also look at appropriate metadata to describe such material and the protection of intellectual property rights (IPR). The University of the Arts, the University College for the Creative Arts and Winchester School of Art worked with their research and practitioner communities in a user-led approach involving questionnaires and interviews. The School of Electronics and Computer Science at the University of Southampton provided the expertise in repository development and the Visual Arts Data Service (VADS) in IPR and copyright. 


\section{Rationale}

IRs have traditionally concentrated on the storage of textual material and this might go some way to explain why the scientific community have tended to have a greater presence in them. Only $30 \%$ of artsbased research outputs submitted to the Research Assessment Exercise in 2008[1] were text-based, the rest took a different form and could be performance pieces, compositions, artefacts, time-based and interactive media or records of exhibitions. Indeed, art practice as research provides a different model to science-based disciplines; here images (or sounds) become the primary medium and text a complementary tool. IRs were not necessarily designed to support this configuration which has perhaps deterred some practice-based researchers from publishing their work in this way.

There are further issues to do with the mapping and documentation of practice-based art research which may also have served to discourage researchers. Some who we interviewed as part of the KULTUR project were broadly supportive of the open access agenda and used sites such as Axis and Artdoxa to promote their work online. However, many others identified barriers to depositing their work in an online repository: doubts about the quality of reproduction, the repository's ability to capture the size and scale of the documentation, whether collaborative works could be shared as well as complex issues of copyright [2].

The KULTUR project was set up in part to explore these barriers and to look at how they could be overcome, the overarching aim being to encourage a greater level of engagement with IRs within the higher education arts sector. A demonstrator model was created for the project and this served as a prototype of an IR for the creative arts through which issues relating to storage, metadata and copyright could be tested and examined. Following the end of the project this demonstrator was split to create two new repositories for the University of the Arts and University of the Creative Arts and was developed as an 'add on' package to the University of Southampton's existing EPrints system.

\section{Metadata}

One of the project's key objectives was to develop metadata that would enable the IR to capture and describe visual works with depth and accuracy. We needed for example to look at using metadata to facilitate the description of multiple items/documents within a single record and to document collaborative works. The metadata schema that resulted offers a 'best practice' model for repository managers as they seek to shape their resource to accommodate research outputs for art and design[3]. In other words, the interoperability shared between the repositories at the University of Southampton, University of the Arts and University College for the Creative Arts has the 
potential to be expanded out to ensure uniformity across the higher education arts sector.

The process of metadata selection involved a number of user-centred activities - online surveys, one-to-one interviews and usability tests and these enabled us to test and refine metadata by providing an insight into how art researchers seek to map and define their work. To give an example, a 'project or series' field was created when it became apparent that there was a lot of project work activity taking place within the creative arts. This enabled relational links to be made between different works if they formed part of the same project.

For metadata to be useful it has to be standardised but this inevitably conflicts with the need for users to have the freedom to describe their work in a meaningful way and not feel constrained by the format. It soon became apparent that there was a tension between the need to develop metadata that was both flexible and consistent and finding a balance here was one of the challenges of the project.

\section{Digital Surrogacy}

Most of the outputs used to populate the KULTUR database were digital surrogates of original works. 'Born digital' works were also added (see Randal Cooke's Light Cadmium Shift, below) and, notwithstanding some technical issues, these items were easy to adapt to the repository environment. Translating a non-digital piece of practice-based art into a digital surrogate was less straightforward however, and this raised fundamental issues about the limits of representing this kind of work within an online repository.

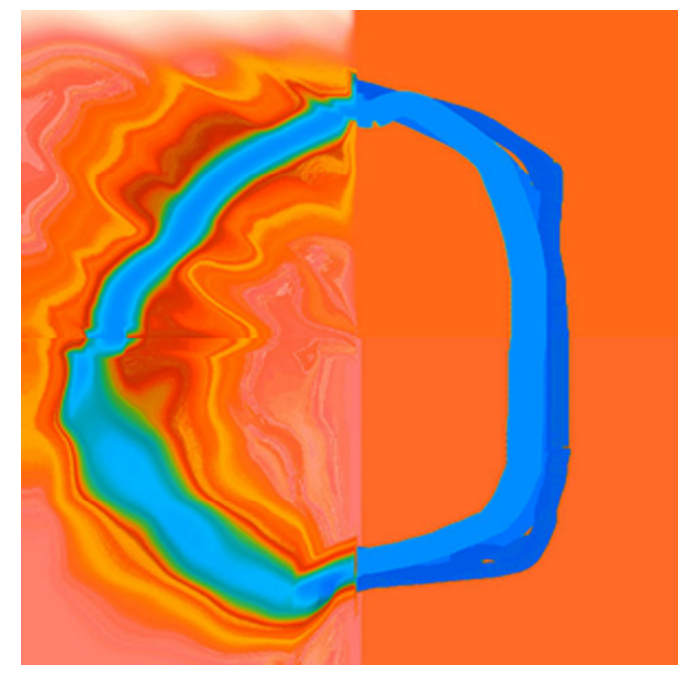

Randal Cooke, Light Cadmium Shift. Born-digital work held in University of the Creative Arts Research Online C Randal Cooke

Gollifer describes art research as process which 'provides modes of inquiry, reflection and production through visual thinking and 
making'[4]. The project had to consider how the IR could convey the knowledge and experiential learning that were embodied in a piece of work. Issues with regard to the quality of representation had to be addressed too. How do we for example capture the 'live' nature of a recorded performance piece or provide a sense of the size of a largescale installation?

These issues were not easy to resolve. One partial solution was to add context, achieved through the inclusion of accompanying textual material - reviews, exhibition guides, web links, etc. - which provided a more rounded picture of the work.

\section{Advocacy}

With so much research activity taking place within the creative arts, an IR presents an opportunity for that research to be stored, shared and promoted both across an institution and to the wider community beyond. It became clear that researchers were greatly interested in how they could use their presence in an IR as a promotional tool, not only to increase the visibility of their work but also to raise their research profile and perhaps attract invitations of work from galleries and other potential partners or collaborators.

Nineteen per cent of the researchers who took part in the project's user survey reported that they did not disseminate their work online[2]. Here the IR can provide researchers with a digital record of their work where none perhaps existed before. This, along with the advantages of visibility previously discussed, offers the benefits of storage and preservation and creates an object which, amongst other things, can be used for teaching purposes or could act as supporting evidence for a funding application.

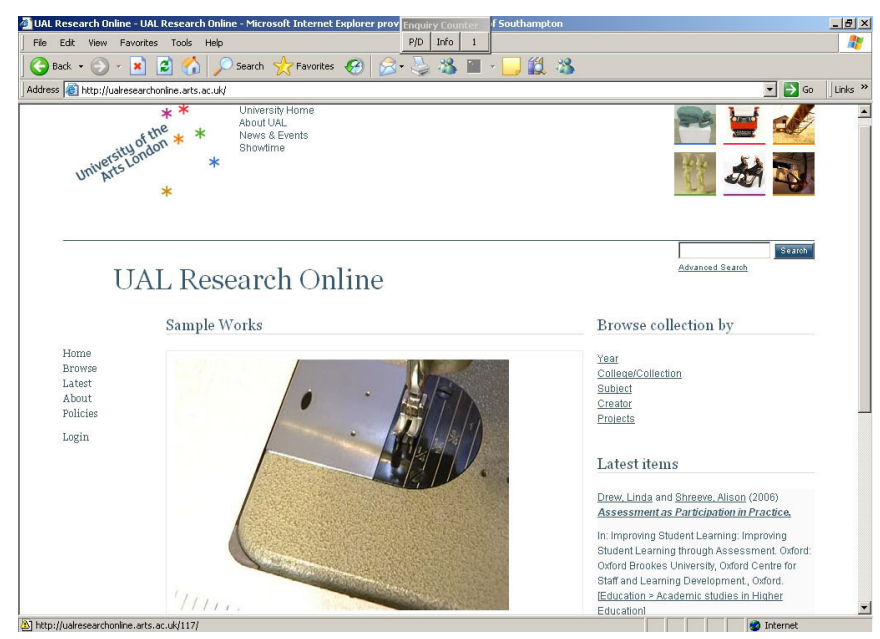

UAL Research Online's homepage: http://ualresearchonline.arts.ac.uk/. Image courtesy of University of the Arts London.

It was important to develop the visual impact of the repository which, as the survey suggested, was a concern for some art researchers. 
These are of course a community with a keen visual sense and there was a feeling amongst them that a badly-designed resource would be a poor showcase for their work; the creation of a professional-looking and visually arresting layout should attract end-users too and identify the repository as a credible resource. The design of the KULTUR demonstrator evolved throughout the project; the figure above illustrates how it made strong use of the visual material collected by the repository with text kept to a minimum.

\section{Sharing and Safeguarding Work}

One of the key areas that the project wanted to address was the tension between sharing work widely and protecting IPR. Much of the impetus for the development of IRs has come from the open access agenda. It has been a condition of JISC funding for repository start up and enhancement that open access to work is maximized and Research Councils now require open access copies of research outputs to be made available. The questionnaire and interviews conducted with researchers helped explore this issue through the creative lifecycle, from the initiation of the work through to dissemination. This placed IPR issues at the heart of the creative process.

Most researchers worked with a variety of media and common activities were installation, photography, video, site specific work, design and digital art. They were often engaging with a large volume of material at different stages of the process as well as a range of formats. A significant number of researchers were engaged in interdisciplinary work, mainly across the arts and humanities, but with some links to science. It is not surprising that this was coupled with a commitment to collaborative work. This included large-scale research projects with shared activity and goals, but also frequent use of specific collaborators with specific skills for a particular purpose. Andrew Gray, who conducted some of the interviews, has talked about the importance of this complex pattern of involvement and responsibility [5]. Not only is there a sophisticated web of multiple rights holders, there is a rich network of relationships in terms of roles and contributions. This showed that it was important for the project to provide a facility to place information about rights holders alongside each individual image or output, as well as express the relationship between individual items, the work and the dissemination of the work. One example of this comprises images showing the creation of a sculpture, a video showing the sculpture, images of an exhibition with the sculpture and other work.

Over $30 \%$ of researchers rated their knowledge of copyright as high or very high and almost $50 \%$ as moderate, showing that the majority felt reasonably confident about copyright issues. Knowledge was highest amongst those who already had work online. This could indicate that uncertainty about copyright is a barrier to placing work online and that the process of engaging with web-based dissemination can improve understanding of the legal context. Almost half indicated a degree of 
uncertainty about how their work would be used by others if it was placed online and a third was unsure of some aspects of copyright ownership.[2] It was therefore important for the project to address these issues both through the design and functionality of the repository and through policy development.

Several tools were built into the repository workflow to provide safeguards to protect IPR and manage copyright concerns. Whilst the aim was to promote as much work as possible in the public domain, it was recognised that a framework of access management was needed to secure the trust of the arts community. It was important to have an option to allow previews but not downloads for some works. A facility to automatically create lower resolution images for display reduced the risk of commercial exploitation of material. Masters of high quality images and video could be stored for preservation purposes and a 'request button' meant that users could ask for copies of high quality or restricted works, giving the artist more control over dissemination. Some researchers found the repository useful to showcase sample images and stills without displaying the whole work.

The project also conducted a review of the IPR policies and processes of the partner institutions, identifying items of good practice and gaps in support. This was led by VADS with their considerable expertise in IPR issues for digitized and hosted non-textual content. As a result some guides to support researchers were developed[6]. To maximize relevance a series of scenarios were produced which aimed to capture the complexity of real situations, whilst reflecting some common issues. This direct support for researchers was supplemented by recommendations for good institutional policy and practice covering four main areas. It is important to develop robust policies and guidance with clear terms of use, rights clearance processes, agreements at the point of deposit and 'take down' policies in case of infringement. The organizational structure needs to support effective resolution of enquiries involving staff from a number of different services, and the organizational culture needs to embrace the routine absorption of current IPR developments. The project partners found that there were a number of key services in each institution that needed to communicate well to develop this overarching IPR support framework, in particular the Library, IT support, legal services and research offices. It was also recommended that the repository support the licensing of material through Creative Commons to clarify terms of reuse[7]. Fully embedding the IPR support into the institutional networks is a challenge and the project partners are still working hard on influencing institutional culture and informing senior managers. 


\section{Future Developments}

The KULTUR project was very much a collaborative venture which enabled the partners involved to share their knowledge, practices and technical expertise and also engage with the range of research activities that were taking place across each institution. It revealed the extent to which IRs can be used to support networking within the creative arts community and pointed to the potential benefits of extending the partnership to include other members of the HE art community. Indeed, researchers who used the KULTUR demonstrator reported that they appreciated being able to search in a shared repository environment which suggests that this is a model that could be further expanded upon.

Project workshops included discussion about the role of the KULTUR model as a tool for research evaluation. Once the Research Excellence Framework (REF) requirements for Art \& Design subjects become known[8], there is the potential to examine how IRs can be used most effectively to store information for submission to the REF exercise and help realise the full impact of research.

There is also the potential to improve usability through integration with additional web 2.0 technologies, like advanced and contextual tagging. Semantic web 3.0 developments could use linked data to improve the presentation of complex relationships between objects and representations and between individual works and collective display.

\section{References}

1. The Research Assessment Exercise 2008 was the latest in a series of peer review evaluations of the quality of research in UK higher education institutions.

2. Sheppard, V. KULTUR project: user survey report. Kultur Project 2009 Available from:

http://kultur.eprints.org/docs/Survey\%20report\%20final\%20Aug \%2008.pdf.

3. Sheppard, V. Metadata report. KULTUR Project 2009; Available from: http://kultur.eprints.org/Metadata\%20report\%20Final.pdf.

4. Gollifer, S. Overview of seminar findings for the e-science scoping study in the visual arts. 2006; Available from: http://ahds.ac.uk/e-science/documents/Gollifer-report.pdf.

5. Gray, A. Institutional Repositories for Creative and Applied Arts Research: The Kultur Project. Ariadne 2009 60]; Available from: http://www.ariadne.ac.uk/issue60/gray/.

6. Eadie, M. 'Scenario A': artist's video with copyright material in the soundtrack. 2009; Available from: http://kultur.eprints.org/docs/IPR\%20scenario\%20A.pdf. 
7. Eadie, M. Fair dealing in an institutional repository: a report for KULTUR. 2008; Available from:

http://kultur.eprints.org/docs/IP\%20paper\%202\%20final.pdf.

8. The Research Excellence Framework (REF) is the new system for assessing the quality of research in the UK higher education institutions. Available from:

http://www.hefce.ac.uk/research/ref. 